\title{
Copula based Measures of Repairable Parallel System with Fault Coverage
}

\author{
Vaishali Tyagi \\ Department of Mathematics and Statistics, \\ Kanya Gurukul Campus (Haridwar), \\ Gurukul Kangri (Deemed to be University), Haridwar, Uttarakhand, India. \\ Corresponding author: vash.tyagi@gmail.com \\ Ritu Arora \\ Department of Mathematics and Statistics, \\ Kanya Gurukul Campus (Haridwar), \\ Gurukul Kangri (Deemed to be University), Haridwar, Uttarakhand, India. \\ E-mail: ritu.arora29@gmail.com \\ Mangey Ram \\ Department of Mathematics, Computer Science \& Engineering, \\ Graphic Era Deemed to be University, Dehradun, Uttarakhand, India. \\ E-mail: mangeyram@geu.ac.in \\ Ioannis S. Triantafyllou \\ Department of Computer Science \& Biomedical Informatics, \\ University of Thessaly, Lamia, Greece. \\ E-mail: itriantafyllou@uth.gr
}

(Received May 21, 2020; Accepted August 11, 2020)

\begin{abstract}
The main objective of this study is to analyse the reliability behaviour of parallel systems with three types of failure, namely unit failure, human failure and major failure. For this purpose, we apply three different statistical techniques, namely copula, coverage and copula-coverage. More precisely, this study presents a stochastic model for analysing the behaviour of a multi-state system consisting of two non-identical units by incorporating the concept of coverage factor and two types of repair facilities between failed state to a normal state. The system could be characterized as being in a failed state due to unit failures, human failure and major failures, such as catastrophic and environmental failure. All failure rates are constant and it is assumed that these are exponentially distributed whereas, repair rates follow the Gumbel-Hougaard copula distribution. The entire system is modelled as a finite-state Markov process. Time-dependent reliability measures like availability, reliability and mean time to failure (MTTF) are obtained by supplementary variable techniques and Laplace transformations. The present study provides a comparative analysis for reliability measures among the aforementioned techniques, while a discussion referring to which technique makes the system more reliable is also developed. Furthermore, numerical simulations are presented to validate the analytical results.
\end{abstract}

Keywords- Perfect coverage, Gumbel-Hougaard copula, Parallel system, Human failure, MTTF, Markov process.

\section{Introduction}

A manufacturer designs the system keeping in mind some objectives to deliver good services. However, after a certain time or due to environmental conditions the system may not perform as well as the manufacturer expected and consequently, the desired result may not be achieved and the system could be then treated as unreliable. Therefore, a reliable system is one that performs 
International Journal of Mathematical, Engineering and Management Sciences

Vol. 6, No. 1, 322-344, 2021

https://doi.org/10.33889/IJMEMS.2021.6.1.021

without any interruption in a specific time under specified conditions. Nowadays, the system's reliability is featured as the main interest of every system design engineer and consequently the way in which the system becomes more reliable, is a big deal. In the field of advanced research in Reliability Theory, many techniques which tend to improve the system's reliability have been introduced and studied. Many researchers implemented copula and coverage techniques to improve the reliability of the underlying system.

Generally speaking, a copula is the function that connects multivariate distribution functions into one-dimensional marginal distribution. In the area of copula distribution, a lot of work has been done by many researchers (Nelsen, 2003; de Melo Mendes and de Souza, 2004; Hu, 2006; Genest and Favre, 2007). A special case of copula distribution is the so-called Gumbel-Hougaard copula distribution, which simply connects bivariate distribution functions into their one-dimensional marginal distribution. Many researchers (Niwas and Garg, 2018; Arora et al., 2020) applied a single type of repair between two adjacent transition states. However, many real-life situations call for two or more types of repair between two transition states. In engineering systems, the GumbelHougaard copula distribution is used when two types of repair are possible between two transition states. Singh and Rawal (2011) analysed a two-unit series system with a controller under the Gumbel-Hougaard copula distribution. The automatic switch-controlled plays a vital role to improve the reliability of the system. Therefore, a controller is used for the proper functioning of the system. Ram and Singh (2012) analysed the cost-benefit of a parallel redundant system under the first come first serve policy (head-of-line repair policy) using the Gumbel-Hougaard family copula and obtained various reliability characteristics like state transition probabilities, mean time to failure and cost-benefit of the system. Dalah and Singh (2014) studied a complex repairable system under the auto-cut switch concept using Gumbel-Hougaard copula distribution and discussed the availability, MTTF and sensitivity of reliability. Ram et al. (2013) discussed waiting for repair concept in a standby system with two types of repair policies namely general and GumbelHougaard family copula and developed the reliability characteristics using the Markov process and supplementary variable techniques. Tyagi et al. (2019) developed a Markovian model having two units in a series configuration in which one unit follow 2-out-of-3: F policy and another has one unit in standby, under human failure and catastrophic failure. El-Sherbeny (2013) studied on a parallel system having two-non identical units with four types of failures and two types of repairs and used graphical evaluation and review technique (GERT) to calculate the availability, mean time to system failure and mean time to replacement. Chopra and Ram (2019) used copula distribution to calculate the availability, reliability, MTTF and profit analysis of parallel systems consisting of two non-identical units with three types of failures namely common cause failure, human failure, and unit failure.

Most of the systems have perfect coverage, but in many situations, the repair is not perfect and the system goes to failed state due to imperfect coverage. Perfect coverage means that the system gets automatically recover from the fault (Manglik and Ram, 2014). Levitin and Amari (2007) evaluated the reliability indices of the systems with multi fault coverage using the universal generating function (UGF) technique. Prabhudeva and Verma (2007) developed a general coverage model (GCM) with hardware failure. GCM helps to perform a failure mode effect analysis of a complex system. Wang et al. (2012) studied the system having a warm standby unit with imperfect coverage. Here, the researcher assumed that both active and standby units have different coverage factors and compared the system with five repair distributions namely exponential, gamma, uniform, normal and deterministic. The mean time to failure (MTTF) of a high voltage (HV) system having power supply and two transmitters have been calculated (Pham,1992) under the concept of dependent 
International Journal of Mathematical, Engineering and Management Sciences

Vol. 6, No. 1, 322-344, 2021

https://doi.org/10.33889/IJMEMS.2021.6.1.021

failure rate and imperfect coverage. Ram and Goyal (2018) studied on flexible manufacturing system under a combinatorial copula-coverage approach and have shown that the mixed copulacoverage technique improves the reliability of the system.

In the past, many researchers have studied the Gumbel-Hougaard copula, the fault coverage, and both techniques, but the copula-coverage technique with perfect coverage has not been covered yet. Therefore, in the present study three techniques namely copula, coverage and copula-coverage with perfect coverage have been discussed. The proposed model consists of two dissimilar units in a parallel configuration. The system gets degraded states due to failure of any one of the units and failed states due to both unit's failure, human failure, and major failure. Further, the effect of these techniques on reliability measures has been analysed. In the context of the copula, the GumbelHougaard family has been used. The main objective of this study is to present a comparative analysis to identify which technique is better to improve system's reliability.

The present paper is organized as follows: Section 2 gives a brief introduction of copula and coverage techniques. Section 3 presents assumptions associated with the model, mathematical model details containing notations and structure of the model. Section 4 describes the formation and solution of the model. Numerical calculations such as availability, reliability, and MTTF have been obtained in Section 5. Section 6 discussed the result of this work. Conclusion of the present paper and the direction of future work is presented at length in Section 7.

\section{Brief Introduction of Copula and Coverage}

The term copula was first introduced by Sklar (1973). Copulas are the functions that connect multivariate distributions functions to their one-dimensional marginal distribution functions (Nelsen, 2007). If $F$ is $m$-dimensional cumulative distribution function with one-dimensional margins $F_{1}, F_{2}, \ldots \ldots \ldots, F_{\mathrm{m}}$ then there exists an $m$ - dimensional copula $C$ such that

where,

$$
F\left(y_{1}, \ldots \ldots, y_{m}\right)=C\left(F_{1}\left(y_{1}\right), \ldots \ldots . . . F_{m}\left(y_{m}\right)\right)
$$

An $m$-dimensional copula is a function such that

$$
C:[0,1]^{m} \longrightarrow[0,1]
$$

The copula approach requires the specification of the marginal distribution of each random variable along with a function that connects them. Patton (2009) has given the idea of conditional copula and applied it on the time-series based model. Copulas are enabled to structure flexible multivariate distributions appearing rich patterns in tail behaviour, different kinds of asymmetry and varying from tail independence to tail dependence (Rodriguez, 2007; Trivedi and Zimmer, 2007).

Gumbel-Hougaard family copula

Gumbel-Hougaard copula is defined as

$$
C\left(u_{1}, u_{2} ; \theta\right)=\exp \left(-\left(\sum_{i=1}^{2}\left(-\log \left(u_{i}\right)\right)^{\theta}\right)^{1 / \theta}\right)
$$

where, $\theta$ is the dependence parameter and $\theta \in[1, \infty)$.

The value of $\theta=1$ corresponds to independence copula and $\theta$ tends to $+\infty$ correspond to the comonotonicity copula. 
International Journal of Mathematical, Engineering and Management Sciences

Vol. 6, No. 1, 322-344, 2021

https://doi.org/10.33889/IJMEMS.2021.6.1.021

Gumbel-Hougaard copula does not allow negative dependence. If $u_{1}=e^{x}$ and $u_{2}=\mu(x)$ then by Gumbel-Hougaard copula the joint probability expression is given by $\exp \left[x^{\theta}+\{\log \mu(x)\}^{\theta}\right]^{1 / \theta}$.

Further, the concept of coverage has introduced by Arnold (1973). Coverage is a technique used to improve the reliability of the system. Coverage is defined as

$$
\text { coverage factor }=\frac{\text { System recover from fault }}{\text { Total fault occur in the system }}
$$

Coverage is the probability of automatic recovery of the system from the fault that occurred during system operation. Coverage provides a more accurate picture of system behaviour and gave more persuasion in reliability estimates. If the value of the coverage factor is equal to one, it is called perfect coverage. Perfect coverage means that the system is able to detect and remove the failure of the redundant system (Manglik and Ram, 2014). Pham (1992) studied a high voltage system with imperfect coverage and constant fault coverage. Levitin and Amari (2007) evaluated reliability characteristics of fault tolerance systems with multi fault coverage using the universal generating function technique. Reibman and Zaretsky (1990) presented a reliability model for network reconfiguration system using fault coverage in a high-reliability model and improve the network's reconfiguration capabilities.

\section{Assumptions, Notations and System Description}

This study provides a comparative analysis among three techniques namely copula, coverage and copula-coverage technique. The assumptions of the proposed model are as follows:

(i) The system consists of two non-identical units in a parallel configuration.

(ii) Initially, the units and the system are in good condition.

(iii) The system contains three states of functioning, namely good, degraded and failed.

(iv) The system could fail due to both unit failure, human failure and major failures, such as a catastrophic failure and environmental failure.

(v) All failure rates are assumed to be constant and follow the Exponential distribution.

(vi) The repair rates follow the Gumbel-Hougaard copula distribution.

(vii) Only a complete state has been repaired and in completely failed states, the system has been repaired by two repair facilities i.e., the Gumbel-Hougaard family of the copula.

(viii) Once the system is repaired, it works like a new one.

The system contains two non- identical units in a parallel configuration. At the beginning, the system is supposed to be in good working condition, say state $S_{0}$. On failure of any one of the two units, another unit takes the load and the system goes to degraded state say state $S_{1}$ and $S_{2}$. The system is said to be in a completely failed state $\left(\mathrm{S}_{3}, \mathrm{~S}_{4}, \mathrm{~S}_{5}, \mathrm{~S}_{6}\right)$ on the failure of both the units, human failure and major failures like a catastrophic failure, electronic failure and environmental failure in the system. The system has two types of repair facilities between completely failed states $\left(\mathrm{S}_{3}, \mathrm{~S}_{4}\right.$, $\mathrm{S}_{5}, \mathrm{~S}_{6}$ ) and original state $\mathrm{S}_{0}$. In such a system, detected faults have been perfectly covered with coverage factor $c$. All failure rates are taken to be constant and follow the exponential distribution. However, repair rates from the state $\left(\mathrm{S}_{3}, \mathrm{~S}_{4}, \mathrm{~S}_{5}, \mathrm{~S}_{6}\right)$ to state $\mathrm{S}_{0}$ follow the Gumbel-Hougaard family of the copula. The whole system is analysed under three different technique namely copula, coverage and copula-coverage to improve the reliability of the system. The description of the transition states associated with the model are given as 
International Journal of Mathematical, Engineering and Management Sciences

Vol. 6, No. 1, 322-344, 2021

https://doi.org/10.33889/IJMEMS.2021.6.1.021

$\mathrm{S}_{0}$ : The system is in good working condition as both the units operate well.

$S_{1}$ : The first unit fails, the second unit takes the load of the first unit, the system is in a degraded state.

$\mathrm{S}_{2}$ : The second unit fails, the system works with the first unit, degraded state of the system.

$\mathrm{S}_{3}$ : Completely failed state due to failure of the second unit after first unit failure.

$\mathrm{S}_{4}$ : Completely failed state due to failure of the first unit after second unit failure.

$\mathrm{S}_{5}$ : Completely failed state due to human failure.

$\mathrm{S}_{6}$ : Completely failed state due to major failure in the system.

Furthermore, some notations used throughout the lines of the present paper are displayed in Table 1.

Table 1. Notations.

\begin{tabular}{ll}
\hline$t$ & Time scale \\
$s$ & Laplace transform variable \\
$x$ & Supplementary variable \\
$\lambda_{1} / \lambda_{2} / \lambda_{h} / \lambda_{m} / c$ & Failure rate of first unit / Failure rate of second unit / human failure rate/ major failure rate/ perfect \\
& coverage parameter. \\
$p_{i}(t)$ & Probability of the state $\mathrm{S}_{i}, i=0,1,2$ \\
$p_{i}(x, t)$ & Probability of state $\mathrm{S}_{i}$ at time $t$ and elapsed repair time $x, i=3,4, h, m$ \\
$\bar{p}(s)$ & Laplace transformation of $p(t)$ \\
$C\left(u_{1}, u_{2}\right)$ & Joint probability for repair rate from a complete failed state to normal state according to Gumbel- \\
& Hougaard family of the copula, where $u_{1}=e^{x}$ and $u_{2}=\mu(x)$ \\
\hline
\end{tabular}

Given the abovementioned assumptions of the proposed model, a transition diagram is developed as shown in Figure 1.

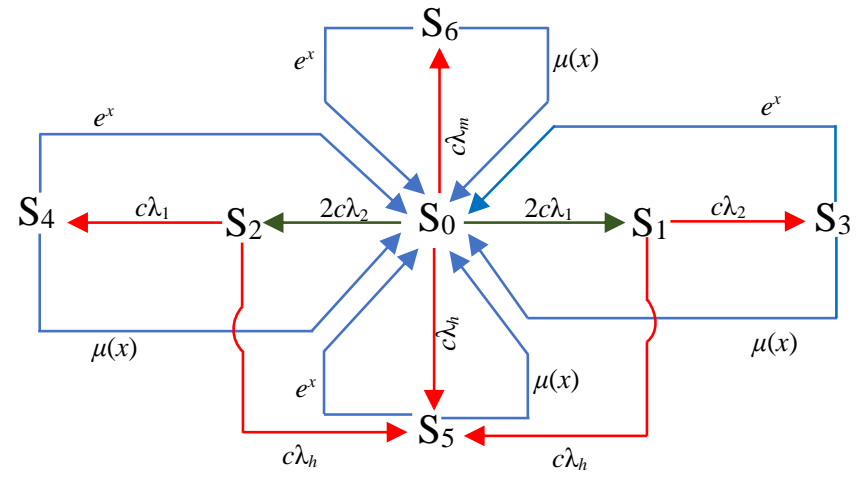

Figure 1. System state transition diagram.

\section{Mathematical Modelling of the Proposed Method}

This section gives the states transition probabilities associated with the model, these probabilities are further used to evaluate the reliability characteristics such as availability, reliability and MTTF. From the state transition diagram, the following differential equations have been derived. 
International Journal of Mathematical, Engineering and Management Sciences

Vol. 6, No. 1, 322-344, 2021

https://doi.org/10.33889/IJMEMS.2021.6.1.021

$\left[\frac{\partial}{\partial t}+2 c \lambda_{1}+2 c \lambda_{2}+c \lambda_{m}+c \lambda_{h}\right] p_{0}(t)=\int_{0}^{\infty} \sum_{i=3,4, h, m} p_{i}(x, t) \exp \left[x^{\theta}+\{\log \mu(x)\}^{\theta}\right]^{1 / \theta} d x$

$\left[\frac{\partial}{\partial t}+c \lambda_{2}+c \lambda_{h}\right] p_{1}(t)=2 c \lambda_{1} p_{0}(t)$

$\left[\frac{\partial}{\partial t}+c \lambda_{1}+c \lambda_{h}\right] p_{2}(t)=2 c \lambda_{2} p_{0}(t)$

$\left[\frac{\partial}{\partial t}+\frac{\partial}{\partial x}+\exp \left[x^{\theta}+\{\log \mu(x)\}^{\theta}\right]^{1 / \theta}\right] p_{i}(x, t)=0, \quad i=3,4, h, m$

Boundary conditions

$$
\begin{aligned}
& p_{3}(0, t)=c \lambda_{2} p_{1}(t) \\
& p_{4}(0, t)=c \lambda_{1} p_{2}(t) \\
& p_{h}(0, t)=c \lambda_{h} p_{0}(t)+c \lambda_{h} p_{1}(t)+c \lambda_{h} p_{2}(t) \\
& p_{m}(0, t)=c \lambda_{m} p_{0}(t)
\end{aligned}
$$

Initial condition

$$
p_{i}(0)=\left\{\begin{array}{ll}
1 & , i=0 \\
0 & , \text { otherwise }
\end{array}\right\}
$$

Taking Laplace transformation from equation (1) to (9), we get the following equations

$$
\begin{aligned}
& {\left[s+2 c \lambda_{1}+2 c \lambda_{2}+c \lambda_{m}+c \lambda_{h}\right] \bar{p}_{0}(s)=1+\int_{0}^{\infty} \sum_{i=3,4, h, m} \bar{p}_{i}(x, s) \exp \left[x^{\theta}+\{\log \mu(x)\}^{\theta}\right]^{1 / \theta} d x} \\
& {\left[s+c \lambda_{2}+c \lambda_{h}\right] \bar{p}_{1}(s)=2 c \lambda_{1} \bar{p}_{0}(s)} \\
& {\left[s+c \lambda_{1}+c \lambda_{h}\right] \bar{p}_{2}(s)=2 c \lambda_{2} \bar{p}_{0}(s)} \\
& {\left[s+\frac{\partial}{\partial x}+\exp \left[x^{\theta}+\{\log \mu(x)\}^{\theta}\right]^{1 / \theta}\right] \bar{p}_{i}(x, s)=0, \quad i=3,4, h, m} \\
& \bar{p}_{3}(0, s)=c \lambda_{2} \bar{p}_{1}(s) \\
& \bar{p}_{4}(0, s)=c \lambda_{1} \bar{p}_{2}(s) \\
& \bar{p}_{h}(0, s)=c \lambda_{h}\left[\bar{p}_{0}(s)+\bar{p}_{1}(s)+\bar{p}_{2}(s)\right] \\
& \bar{p}_{m}(0, s)=c \lambda_{m} \bar{p}_{0}(s)
\end{aligned}
$$


After solving the Equations (10) - (13) by the aid of (14) - (17), one can get the state transition probabilities as

$$
\bar{p}_{0}(s)=\frac{1}{\left(s+2 c \lambda_{1}+2 c \lambda_{2}+c \lambda_{m}+c \lambda_{h}\right)-\bar{S}(s)\left[\begin{array}{l}
\frac{2 c^{2} \lambda_{1} \lambda_{2}}{s+c \lambda_{2}+c \lambda_{h}}+\frac{2 c^{2} \lambda_{1} \lambda_{2}}{s+c \lambda_{1}+c \lambda_{h}}+\frac{2 c^{2} \lambda_{1} \lambda_{h}}{s+c \lambda_{2}+c \lambda_{h}} \\
+\frac{2 c^{2} \lambda_{2} \lambda_{h}}{s+c \lambda_{1}+c \lambda_{h}}+c \lambda_{m}+c \lambda_{h}
\end{array}\right]}
$$

$\bar{p}_{1}(s)=\frac{2 c \lambda_{1} \bar{p}_{0}(s)}{s+c \lambda_{2}+c \lambda_{h}}$

$\bar{p}_{2}(s)=\frac{2 c \lambda_{2} \bar{p}_{0}(s)}{s+c \lambda_{1}+c \lambda_{h}}$

$\bar{p}_{3}(s)=\frac{2 c^{2} \lambda_{1} \lambda_{2} \bar{p}_{0}(s)}{s+c \lambda_{2}+c \lambda_{h}}\left(\frac{1-\bar{S}(s)}{s}\right)$

$\bar{p}_{4}(s)=\frac{2 c^{2} \lambda_{1} \lambda_{2} \bar{p}_{0}(s)}{s+c \lambda_{1}+c \lambda_{h}}\left(\frac{1-\bar{S}(s)}{s}\right)$

$\bar{p}_{h}(s)=\bar{p}_{0}(s)\left(\frac{1-\bar{S}(s)}{s}\right)\left[c \lambda_{h}+\frac{2 c^{2} \lambda_{1} \lambda_{h}}{s+c \lambda_{2}+c \lambda_{h}}+\frac{2 c^{2} \lambda_{2} \lambda_{h}}{s+c \lambda_{1}+c \lambda_{h}}\right]$

$\bar{p}_{m}(s)=c \lambda_{m} \bar{p}_{0}(s)\left(\frac{1-\bar{S}(s)}{s}\right)$

where,

$\bar{S}(s)=\int_{0}^{\infty} \phi(\mathrm{x}) e^{-s x-\int_{0}^{x} \phi(\mathrm{x}) d x} d x$ and $\phi(\mathrm{x})=\exp \left[x^{\theta}+\{\log \mu(x)\}^{\theta}\right]^{1 / \theta}$.

Up and downstate probability are as follow:

$$
\begin{aligned}
\bar{p}_{u p}(s) & =\sum_{i=0}^{2} \bar{p}_{i}(s) \\
& =\bar{p}_{0}(s)\left[1+\frac{2 c \lambda_{1}}{s+c \lambda_{2}+c \lambda_{h}}+\frac{2 c \lambda_{2}}{s+c \lambda_{1}+c \lambda_{h}}\right] \\
\bar{p}_{\text {down }}(s) & =\sum_{i=3,4, h, m} \bar{p}_{i}(s) \\
& =\bar{p}_{0}(s)\left(\frac{1-\bar{S}(s)}{s}\right)\left[\frac{2 c^{2} \lambda_{1} \lambda_{2}}{s+c \lambda_{2}+c \lambda_{h}}+\frac{2 c^{2} \lambda_{1} \lambda_{2}}{s+c \lambda_{1}+c \lambda_{h}}+\frac{2 c^{2} \lambda_{1} \lambda_{h}}{s+c \lambda_{2}+c \lambda_{h}}+\frac{2 c^{2} \lambda_{2} \lambda_{h}}{s+c \lambda_{1}+c \lambda_{h}}+c \lambda_{h}+c \lambda_{m}\right]
\end{aligned}
$$


International Journal of Mathematical, Engineering and Management Sciences

Vol. 6, No. 1, 322-344, 2021

https://doi.org/10.33889/IJMEMS.2021.6.1.021

\section{Numerical Calculations}

In this section, some numerical computations have been accomplished by the aid of the abovementioned differential equations.

\subsection{Availability Analysis}

Generally speaking, availability simply means that the system performs its work in a specified time $t$. Availability is one of the most important reliability measures, since it is associated with both the failure and repair rate of the system. It is always a time function. In this subsection, the availability of the underlying system with different techniques is presented.

\subsubsection{Availability of the System without Copula-Coverage.}

In this case, availability expression is given by

$A_{1}=\left[\frac{1}{s+2 \lambda_{1}+2 \lambda_{2}+\lambda_{m}+\lambda_{h}-\frac{\mu}{\mu+s}\left[\frac{2 \lambda_{1} \lambda_{2}}{s+\lambda_{2}+\lambda_{h}}+\frac{2 \lambda_{1} \lambda_{2}}{s+\lambda_{1}+\lambda_{h}}+\frac{2 \lambda_{1} \lambda_{h}}{s+\lambda_{2}+\lambda_{h}}+\frac{2 \lambda_{2} \lambda_{h}}{s+\lambda_{1}+\lambda_{h}}\right]}\right]\left[1+\frac{2 \lambda_{1}}{s+\lambda_{2}+\lambda_{h}}+\frac{2 \lambda_{2}}{s+\lambda_{1}+\lambda_{h}}\right]$

By setting the values of $\lambda_{1}=0.05, \lambda_{2}=0.10, \lambda_{h}=0.15, \lambda_{m}=0.20$ and $\mu=1$ in Equation (27) and then taking inverse Laplace transform, we may produce several numerical results. Table 2 displays the availability of the system for time $t$ from 0 to 25 .

Table 2. Availability of the system without copula-coverage.

\begin{tabular}{cc}
\hline Time $(t)$ & Availability $P_{u p}(t)$ \\
\hline 1 & 1.000000 \\
2 & 0.818066 \\
3 & 0.780879 \\
4 & 0.776633 \\
5 & 0.778716 \\
6 & 0.781219 \\
7 & 0.783105 \\
8 & 0.784383 \\
9 & 0.785222 \\
10 & 0.785769 \\
11 & 0.786127 \\
12 & 0.786363 \\
13 & 0.786520 \\
14 & 0.786626 \\
15 & 0.786699 \\
16 & 0.786749 \\
17 & 0.786785 \\
18 & 0.786810 \\
19 & 0.786828 \\
20 & 0.786842 \\
21 & 0.786852 \\
22 & 0.786859 \\
23 & 0.786865 \\
24 & 0.786869 \\
25 & 0.786873 \\
\end{tabular}


International Journal of Mathematical, Engineering and Management Sciences

Vol. 6, No. 1, 322-344, 2021

https://doi.org/10.33889/IJMEMS.2021.6.1.021

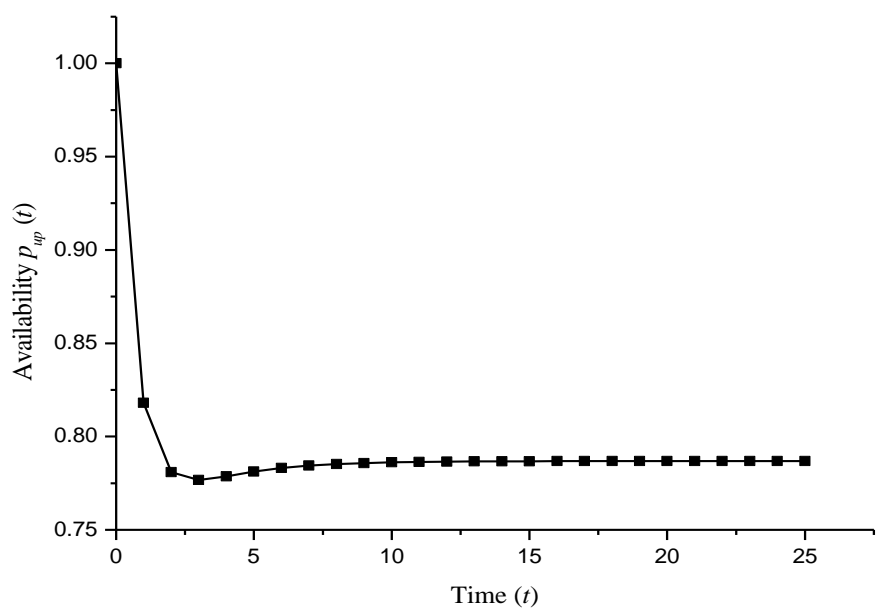

Figure 2. Time dependant availability of the system without copula-coverage.

\subsubsection{Availability of the System with Copula (When Coverage is Ignored).}

In this case, availability of the system is given by putting $x=1, \mu(x)=1$ and $\theta=1$ in Equation (25)

$A_{2}=\left[\frac{1}{s+2 \lambda_{1}+2 \lambda_{2}+\lambda_{m}+\lambda_{h}-\frac{e}{e+s}\left[\frac{2 \lambda_{1} \lambda_{2}}{s+\lambda_{2}+\lambda_{h}}+\frac{2 \lambda_{1} \lambda_{2}}{s+\lambda_{1}+\lambda_{h}}+\frac{2 \lambda_{1} \lambda_{h}}{s+\lambda_{2}+\lambda_{h}}+\frac{2 \lambda_{2} \lambda_{h}}{s+\lambda_{1}+\lambda_{h}}\right]}\right]\left[1+\frac{2 \lambda_{1}}{s+\lambda_{2}+\lambda_{h}}+\frac{2 \lambda_{2}}{s+\lambda_{1}+\lambda_{h}}\right]$

Table 3. Availability of the system with copula.

\begin{tabular}{cc}
\hline Time $(t)$ & Availability $P_{u p}(t)$ \\
\hline 0 & 1.000000 \\
1 & 0.898088 \\
2 & 0.899352 \\
3 & 0.903032 \\
4 & 0.905419 \\
5 & 0.906893 \\
6 & 0.907804 \\
7 & 0.908369 \\
8 & 0.908724 \\
9 & 0.908948 \\
10 & 0.909091 \\
11 & 0.909184 \\
12 & 0.909245 \\
13 & 0.909285 \\
14 & 0.909313 \\
15 & 0.909332 \\
16 & 0.909346 \\
17 & 0.909355 \\
18 & 0.909362 \\
19 & 0.909368 \\
20 & 0.909371 \\
21 & 0.909374 \\
22 & 0.909376 \\
23 & 0.909379 \\
24 & 0.909379 \\
25 & 0.909381 \\
\hline
\end{tabular}


By replacing $\lambda_{1}=0.05, \lambda_{2}=0.10, \lambda_{h}=0.15, \lambda_{m}=0.20$ in the above availability expression and then taking inverse Laplace transform of Equation (28), we may produce several numerical results. Table 3 displays the availability of the underlying system for time $t$ from 0 to 25 .

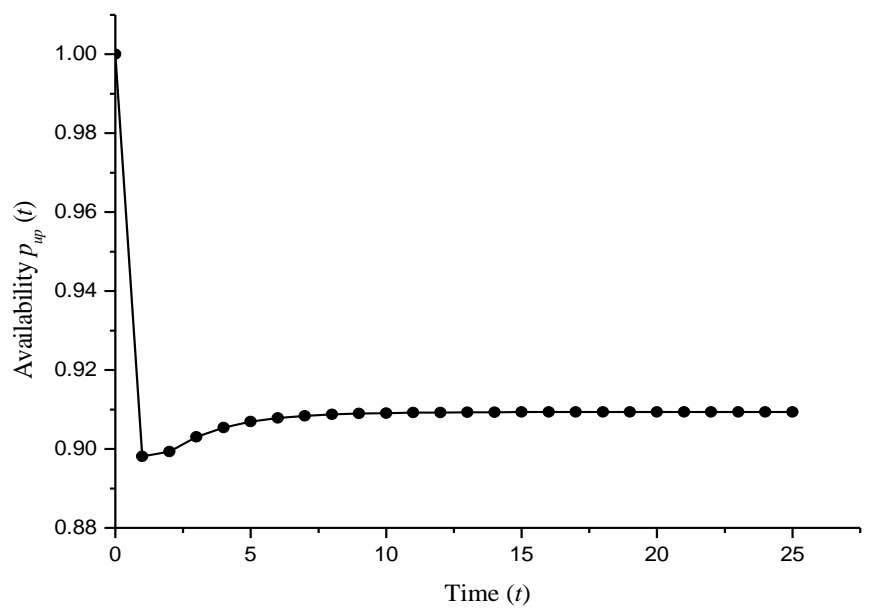

Figure 3. Availability of the system with copula.

\subsubsection{Availability of the System with Coverage (When Copula is Ignored).}

In this case, availability expression is given by putting $\phi(x)=1$ in Equation (25).

Table 4. Availability of the system at a different value of coverage factor.

\begin{tabular}{|c|c|c|c|c|}
\hline \multirow{2}{*}{ Time $(t)$} & \multicolumn{4}{|c|}{ Availability $P_{u p}(t)$} \\
\hline & $c=0.2$ & $c=0.4$ & $c=0.6$ & $c=0.8$ \\
\hline 0 & 1.000000 & 1.000000 & 1.000000 & 1.000000 \\
\hline 1 & 0.957565 & 0.918492 & 0.882423 & 0.849041 \\
\hline 2 & 0.943850 & 0.895220 & 0.852587 & 0.814775 \\
\hline 3 & 0.939892 & 0.889977 & 0.847327 & 0.809983 \\
\hline 4 & 0.939205 & 0.890076 & 0.848402 & 0.811752 \\
\hline 5 & 0.939573 & 0.891556 & 0.850679 & 0.814336 \\
\hline 6 & 0.940246 & 0.893217 & 0.852819 & 0.816484 \\
\hline 7 & 0.940970 & 0.894723 & 0.854554 & 0.818066 \\
\hline 8 & 0.941665 & 0.896006 & 0.855895 & 0.819189 \\
\hline 9 & 0.942308 & 0.897076 & 0.856915 & 0.819976 \\
\hline 10 & 0.942893 & 0.897961 & 0.857688 & 0.820527 \\
\hline 11 & 0.943425 & 0.898691 & 0.857688 & 0.820914 \\
\hline 12 & 0.943907 & 0.899293 & 0.858716 & 0.821186 \\
\hline 13 & 0.944343 & 0.899790 & 0.859053 & 0.821379 \\
\hline 14 & 0.944737 & 0.900200 & 0.859310 & 0.821518 \\
\hline 15 & 0.945095 & 0.900539 & 0.859506 & 0.821618 \\
\hline 16 & 0.945418 & 0.900819 & 0.859657 & 0.821690 \\
\hline 17 & 0.945711 & 0.901052 & 0.859773 & 0.821744 \\
\hline 18 & 0.945976 & 0.901245 & 0.859863 & 0.821783 \\
\hline 19 & 0.946217 & 0.901405 & 0.859933 & 0.821813 \\
\hline 20 & 0.946434 & 0.901537 & 0.859987 & 0.821835 \\
\hline 21 & 0.946632 & 0.901648 & 0.860030 & 0.821852 \\
\hline 22 & 0.946810 & 0.901740 & 0.860064 & 0.821866 \\
\hline 23 & 0.946973 & 0.901817 & 0.860091 & 0.821876 \\
\hline 24 & 0.947119 & 0.901882 & 0.860112 & 0.821884 \\
\hline 25 & 0.947253 & 0.901936 & 0.860129 & 0.821890 \\
\hline
\end{tabular}


International Journal of Mathematical, Engineering and Management Sciences

Vol. 6, No. 1, 322-344, 2021

https://doi.org/10.33889/IJMEMS.2021.6.1.021

$$
\begin{aligned}
A_{3}= & {\left[\frac{1}{s+2 c \lambda_{1}+2 c \lambda_{2}+c \lambda_{m}+c \lambda_{h}-\frac{1}{1+s}\left[\frac{2 c^{2} \lambda_{1} \lambda_{2}}{s+c \lambda_{2}+c \lambda_{h}}+\frac{2 c^{2} \lambda_{1} \lambda_{2}}{s+c \lambda_{1}+c \lambda_{h}}+\frac{2 c^{2} \lambda_{1} \lambda_{h}}{s+c \lambda_{2}+c \lambda_{h}}+\frac{2 c^{2} \lambda_{2} \lambda_{h}}{s+c \lambda_{1}+c \lambda_{h}}\right]}\right] \times } \\
& \quad\left[1+\frac{2 c \lambda_{1}}{s+c \lambda_{2}+c \lambda_{h}}+\frac{2 c \lambda_{2}}{s+c \lambda_{1}+c \lambda_{h}}\right]
\end{aligned}
$$

Further, by putting $\lambda_{1}=0.05, \lambda_{2}=0.10, \lambda_{h}=0.15, \lambda_{m}=0.20$ and then taking the inverse Laplace transform of Equation (29), we may produce several numerical results. Table 4 presents the availability of the system for time $t$ from 0 to 25 at different values of coverage factor.

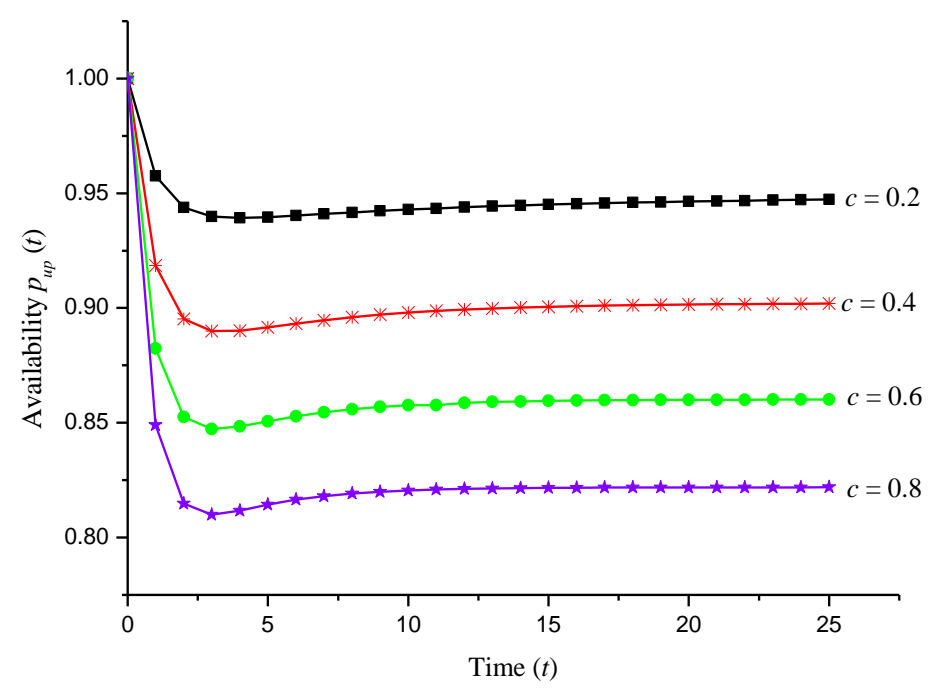

Figure 4. Availability of the system with coverage.

\subsubsection{Availability of the System with Copula- Coverage (Both Copula and Coverage Technique Apply Together)}

By replacing $x=1, \mu(x)=1$ and $\theta=1$ in Equation (25), we get the following availability expression

$$
\begin{aligned}
A_{4}= & {\left[\frac{1}{s+2 c \lambda_{1}+2 c \lambda_{2}+c \lambda_{m}+c \lambda_{h}-\frac{e}{e+s}\left[\frac{2 c^{2} \lambda_{1} \lambda_{2}}{s+c \lambda_{2}+c \lambda_{h}}+\frac{2 c^{2} \lambda_{1} \lambda_{2}}{s+c \lambda_{1}+c \lambda_{h}}+\frac{2 c^{2} \lambda_{1} \lambda_{h}}{s+c \lambda_{2}+c \lambda_{h}}+\frac{2 c^{2} \lambda_{2} \lambda_{h}}{s+c \lambda_{1}+c \lambda_{h}}\right]}\right] \times } \\
& {\left[1+\frac{2 c \lambda_{1}}{s+c \lambda_{2}+c \lambda_{h}}+\frac{2 c \lambda_{2}}{s+c \lambda_{1}+c \lambda_{h}}\right] }
\end{aligned}
$$

We next replace $\lambda_{1}=0.05, \lambda_{2}=0.10, \lambda_{h}=0.15, \lambda_{m}=0.20$. After taking inverse Laplace transform of Equation (30) and varying time $t=0$ to 25 , we get the Table 5 at different values of coverage factor as $c=0.2,0.4,0.6$ and 0.8 . 
International Journal of Mathematical, Engineering and Management Sciences

Vol. 6, No. 1, 322-344, 2021

https://doi.org/10.33889/IJMEMS.2021.6.1.021

Table 5. Availability of the system with copula at different values of coverage factor.

\begin{tabular}{|c|c|c|c|c|}
\hline \multirow{2}{*}{ Time $(t)$} & \multicolumn{4}{|c|}{ Availability $P_{u p}(t)$} \\
\hline & $c=0.2$ & $c=0.4$ & $c=0.6$ & $c=0.8$ \\
\hline 0 & 1.000000 & 1.000000 & 1.000000 & 1.000000 \\
\hline 1 & 0.976797 & 0.955171 & 0.934943 & 0.915959 \\
\hline 2 & 0.975835 & 0.954173 & 0.934464 & 0.916294 \\
\hline 3 & 0.976195 & 0.955397 & 0.936647 & 0.919324 \\
\hline 4 & 0.976596 & 0.956515 & 0.938399 & 0.921501 \\
\hline 5 & 0.976962 & 0.957435 & 0.939705 & 0.922976 \\
\hline 6 & 0.977294 & 0.958188 & 0.940676 & 0.923974 \\
\hline 7 & 0.977594 & 0.958804 & 0.941397 & 0.924650 \\
\hline 8 & 0.977864 & 0.959309 & 0.941934 & 0.925110 \\
\hline 9 & 0.978109 & 0.959722 & 0.942335 & 0.925425 \\
\hline 10 & 0.978330 & 0.960061 & 0.942635 & 0.925642 \\
\hline 11 & 0.978530 & 0.960339 & 0.942859 & 0.925791 \\
\hline 12 & 0.978711 & 0.960568 & 0.943028 & 0.925895 \\
\hline 13 & 0.978874 & 0.960755 & 0.943156 & 0.925969 \\
\hline 14 & 0.979022 & 0.960909 & 0.943252 & 0.926021 \\
\hline 15 & 0.979156 & 0.961037 & 0.943325 & 0.926058 \\
\hline 16 & 0.979276 & 0.961141 & 0.943381 & 0.926085 \\
\hline 17 & 0.979386 & 0.961228 & 0.943424 & 0.926104 \\
\hline 18 & 0.979485 & 0.961299 & 0.943457 & 0.926119 \\
\hline 19 & 0.979574 & 0.961359 & 0.943482 & 0.926129 \\
\hline 20 & 0.979655 & 0.961407 & 0.943502 & 0.926138 \\
\hline 21 & 0.979729 & 0.961448 & 0.943518 & 0.926145 \\
\hline 22 & 0.979795 & 0.961482 & 0.943530 & 0.926149 \\
\hline 23 & 0.979856 & 0.961509 & 0.943539 & 0.926154 \\
\hline 24 & 0.979909 & 0.961533 & 0.943548 & 0.926157 \\
\hline 25 & 0.979959 & 0.961553 & 0.943554 & 0.926159 \\
\hline
\end{tabular}

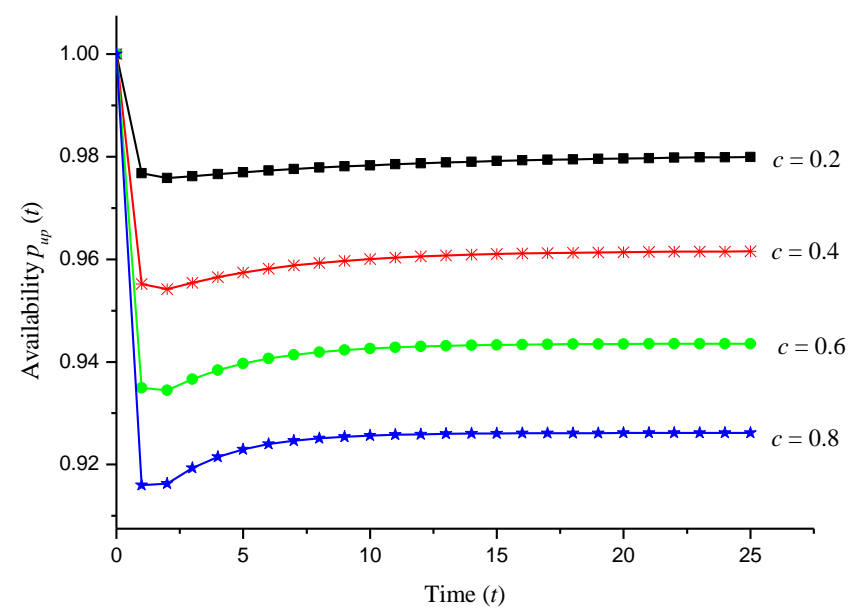

Figure 5. Availability of the system with copula-coverage.

\subsection{Reliability Analysis}

Reliability is a probability that any system works well without any damage within a specific time under the stated condition. The reliability of the system can be found in the case that repair facilities not available. Hence, the reliability of the system can be calculated by putting a repair rate equal to zero. 
International Journal of Mathematical, Engineering and Management Sciences

Vol. 6, No. 1, 322-344, 2021

https://doi.org/10.33889/IJMEMS.2021.6.1.021

\subsubsection{Reliability of the System without Copula- Coverage}

Reliability of the system without copula-coverage is given by

$$
R_{1}=\frac{1}{s+2 \lambda_{1}+2 \lambda_{2}+\lambda_{m}+\lambda_{h}}\left[1+\frac{2 \lambda_{1}}{s+\lambda_{2}+\lambda_{h}}+\frac{2 \lambda_{2}}{s+\lambda_{1}+\lambda_{h}}\right]
$$

We next replace $\lambda_{1}=0.05, \lambda_{2}=0.10, \lambda_{h}=0.15, \lambda_{m}=0.20$ and then take inverse Laplace transform of Equation (31). Table 6 displays the reliability of the system for time $t$ from 0 to 25 .

Table 6. Reliability of the system without copula-coverage.

\begin{tabular}{cc}
\hline Time $(t)$ & Reliability R $(t)$ \\
\hline 0 & 1.000000 \\
1 & 0.718095 \\
2 & 0.532826 \\
3 & 0.405481 \\
4 & 0.314366 \\
5 & 0.246976 \\
6 & 0.195832 \\
7 & 0.156271 \\
8 & 0.125251 \\
9 & 0.100696 \\
10 & 0.081129 \\
11 & 0.065468 \\
12 & 0.052891 \\
13 & 0.042769 \\
14 & 0.034610 \\
15 & 0.028025 \\
16 & 0.022705 \\
17 & 0.018403 \\
18 & 0.014924 \\
19 & 0.012107 \\
20 & 0.009825 \\
21 & 0.007077 \\
22 & 0.006478 \\
23 & 0.005263 \\
24 & 0.004277 \\
25 & 0.003477 \\
\hline
\end{tabular}

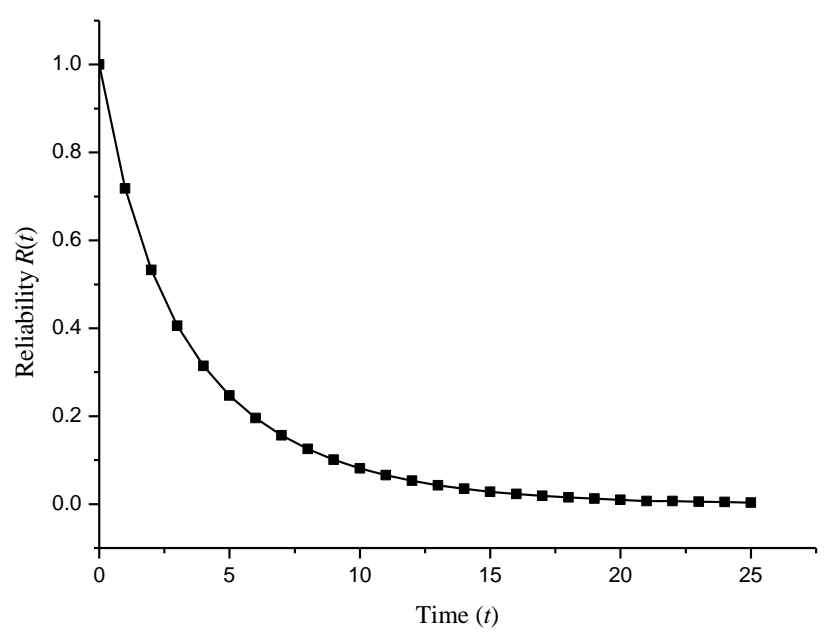

Figure 6. Reliability of the system without copula -coverage. 
International Journal of Mathematical, Engineering and Management Sciences

Vol. 6, No. 1, 322-344, 2021

https://doi.org/10.33889/IJMEMS.2021.6.1.021

\subsubsection{Reliability of the System with Copula}

Ignored coverage factor and put repair rate is equal to zero in Equation (25) to get the reliability expression of the system with copula, given by as follow

$$
R_{1}=\frac{1}{s+2 \lambda_{1}+2 \lambda_{2}+\lambda_{m}+\lambda_{h}}\left[1+\frac{2 \lambda_{1}}{s+\lambda_{2}+\lambda_{h}}+\frac{2 \lambda_{2}}{s+\lambda_{1}+\lambda_{h}}\right]
$$

We first set $\lambda_{1}=0.05, \lambda_{2}=0.10, \lambda_{h}=0.15, \lambda_{m}=0.20$ and then take inverse Laplace transform of Equation (32). Table 7 displays the reliability of the system for time $t$ from 0 to 25 .

Table 7. Reliability of the system with copula.

\begin{tabular}{cc}
\hline Time $(t)$ & Reliability R $(t)$ \\
\hline 0 & 1.000000 \\
1 & 0.718095 \\
2 & 0.532826 \\
3 & 0.405481 \\
4 & 0.314366 \\
5 & 0.246976 \\
6 & 0.195832 \\
7 & 0.156271 \\
8 & 0.125251 \\
9 & 0.100696 \\
10 & 0.081129 \\
11 & 0.065468 \\
12 & 0.052891 \\
13 & 0.042769 \\
14 & 0.034610 \\
15 & 0.028025 \\
16 & 0.022705 \\
17 & 0.018403 \\
18 & 0.014924 \\
19 & 0.012107 \\
20 & 0.009825 \\
21 & 0.007077 \\
22 & 0.006478 \\
23 & 0.005263 \\
24 & 0.004277 \\
25 & 0.003477 \\
\hline
\end{tabular}

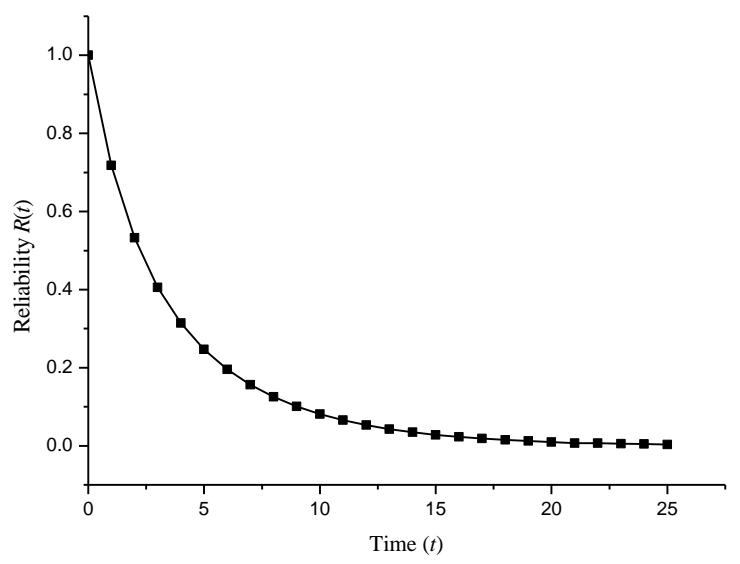

Figure 7. Reliability of the system with copula. 
International Journal of Mathematical, Engineering and Management Sciences

Vol. 6, No. 1, 322-344, 2021

https://doi.org/10.33889/IJMEMS.2021.6.1.021

\subsubsection{Reliability of the System with Coverage}

In this case, the reliability of the system is given by

$$
R_{2}=\frac{1}{s+2 c \lambda_{1}+2 c \lambda_{2}+c \lambda_{m}+c \lambda_{h}}\left[1+\frac{2 c \lambda_{1}}{s+c \lambda_{2}+c \lambda_{h}}+\frac{2 c \lambda_{2}}{s+c \lambda_{1}+c \lambda_{h}}\right]
$$

We first set the values of failure rates $\lambda_{1}=0.05, \lambda_{2}=0.10, \lambda_{h}=0.15, \lambda_{m}=0.20$ and then take inverse Laplace transform of Equation (33). Further, by putting $c=0.2,0.4,0.6,0.8$ and varying $t=0$ to 25 , we get the following table.

Table 8. Reliability of the system with coverage.

\begin{tabular}{|c|c|c|c|c|}
\hline \multirow[t]{2}{*}{ Time $(t)$} & \multicolumn{4}{|c|}{ Reliability $R(t)$} \\
\hline & $c=0.2$ & $c=0.4$ & $c=0.6$ & $c=0.8$ \\
\hline 0 & 1.000000 & 1.000000 & 1.000000 & 1.000000 \\
\hline 1 & 0.933131 & 0.872082 & 0.816242 & 0.765072 \\
\hline 2 & 0.872082 & 0.765072 & 0.674885 & 0.598313 \\
\hline 3 & 0.816242 & 0.674885 & 0.564319 & 0.476426 \\
\hline 4 & 0.765072 & 0.598313 & 0.476426 & 0.384858 \\
\hline 5 & 0.718095 & 0.532826 & 0.405481 & 0.314366 \\
\hline 6 & 0.674885 & 0.476426 & 0.347410 & 0.258966 \\
\hline 7 & 0.635069 & 0.427525 & 0.299284 & 0.214684 \\
\hline 8 & 0.598313 & 0.384858 & 0.258966 & 0.178815 \\
\hline 9 & 0.564319 & 0.347410 & 0.224877 & 0.149461 \\
\hline 10 & 0.532826 & 0.314366 & 0.195832 & 0.125251 \\
\hline 11 & 0.503598 & 0.285065 & 0.170927 & 0.105167 \\
\hline 12 & 0.476426 & 0.258966 & 0.149461 & 0.088434 \\
\hline 13 & 0.451124 & 0.235627 & 0.130882 & 0.074447 \\
\hline 14 & 0.427525 & 0.214684 & 0.114747 & 0.062728 \\
\hline 15 & 0.405481 & 0.195832 & 0.100696 & 0.052891 \\
\hline 16 & 0.384858 & 0.178815 & 0.088434 & 0.044623 \\
\hline 17 & 0.365536 & 0.163419 & 0.077714 & 0.037665 \\
\hline 18 & 0.347410 & 0.149461 & 0.068330 & 0.031806 \\
\hline 19 & 0.330382 & 0.136784 & 0.060106 & 0.026868 \\
\hline 20 & 0.314366 & 0.125251 & 0.052891 & 0.022704 \\
\hline 21 & 0.299284 & 0.114747 & 0.046558 & 0.019192 \\
\hline 22 & 0.285065 & 0.105167 & 0.040994 & 0.016228 \\
\hline 23 & 0.271644 & 0.096423 & 0.036105 & 0.013725 \\
\hline 24 & 0.258966 & 0.088434 & 0.031806 & 0.011611 \\
\hline 25 & 0.246976 & 0.081129 & 0.028025 & 0.009825 \\
\hline
\end{tabular}

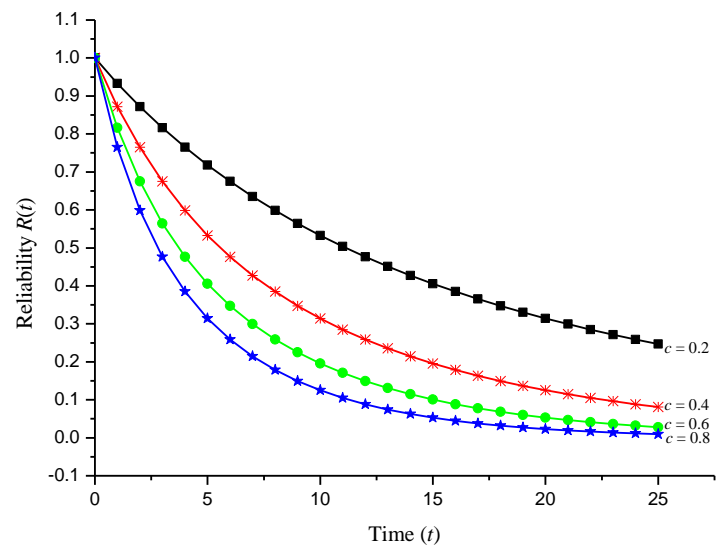

Figure 8. Reliability of the system with coverage. 
International Journal of Mathematical, Engineering and Management Sciences

Vol. 6, No. 1, 322-344, 2021

https://doi.org/10.33889/IJMEMS.2021.6.1.021

\subsubsection{Reliability of the System with Copula-Coverage.}

In this case, reliability expression is given by Equation (33). Now put $\lambda_{1}=0.05, \lambda_{2}=0.10, \lambda_{h}=0.15$, $\lambda_{m}=0.20$ and then take inverse Laplace transform of Equation (33). Further, put $c=0.2,0.4,0.6$, 0.8 and varying $t=0$ to 25 , we get Table 9 as follow.

Table 9. Reliability of the system with copula- coverage.

\begin{tabular}{|c|c|c|c|c|}
\hline \multirow[t]{2}{*}{ Time $(t)$} & \multicolumn{4}{|c|}{ Reliability $R(t)$} \\
\hline & $c=0.2$ & $c=0.4$ & $c=0.6$ & $c=0.8$ \\
\hline 0 & 1.000000 & 1.000000 & 1.000000 & 1.000000 \\
\hline 1 & 0.933131 & 0.872082 & 0.816242 & 0.765072 \\
\hline 2 & 0.872082 & 0.765072 & 0.674885 & 0.598313 \\
\hline 3 & 0.816242 & 0.674885 & 0.564319 & 0.476426 \\
\hline 4 & 0.765072 & 0.598313 & 0.476426 & 0.384858 \\
\hline 5 & 0.718095 & 0.532826 & 0.405481 & 0.314366 \\
\hline 6 & 0.674885 & 0.476426 & 0.347410 & 0.258966 \\
\hline 7 & 0.635069 & 0.427525 & 0.299284 & 0.214684 \\
\hline 8 & 0.598313 & 0.384858 & 0.258966 & 0.178815 \\
\hline 9 & 0.564319 & 0.347410 & 0.224877 & 0.149461 \\
\hline 10 & 0.532826 & 0.314366 & 0.195832 & 0.125251 \\
\hline 11 & 0.503598 & 0.285065 & 0.170927 & 0.105167 \\
\hline 12 & 0.476426 & 0.258966 & 0.149461 & 0.088434 \\
\hline 13 & 0.451124 & 0.235627 & 0.130882 & 0.074447 \\
\hline 14 & 0.427525 & 0.214684 & 0.114747 & 0.062728 \\
\hline 15 & 0.405481 & 0.195832 & 0.100696 & 0.052891 \\
\hline 16 & 0.384858 & 0.178815 & 0.088434 & 0.044623 \\
\hline 17 & 0.365536 & 0.163419 & 0.077714 & 0.037665 \\
\hline 18 & 0.347410 & 0.149461 & 0.068330 & 0.031806 \\
\hline 19 & 0.330382 & 0.136784 & 0.060106 & 0.026868 \\
\hline 20 & 0.314366 & 0.125251 & 0.052891 & 0.022704 \\
\hline 21 & 0.299284 & 0.114747 & 0.046558 & 0.019192 \\
\hline 22 & 0.285065 & 0.105167 & 0.040994 & 0.016228 \\
\hline 23 & 0.271644 & 0.096423 & 0.036105 & 0.013725 \\
\hline 24 & 0.258966 & 0.088434 & 0.031806 & 0.011611 \\
\hline 25 & 0.246976 & 0.081129 & 0.028025 & 0.009825 \\
\hline
\end{tabular}

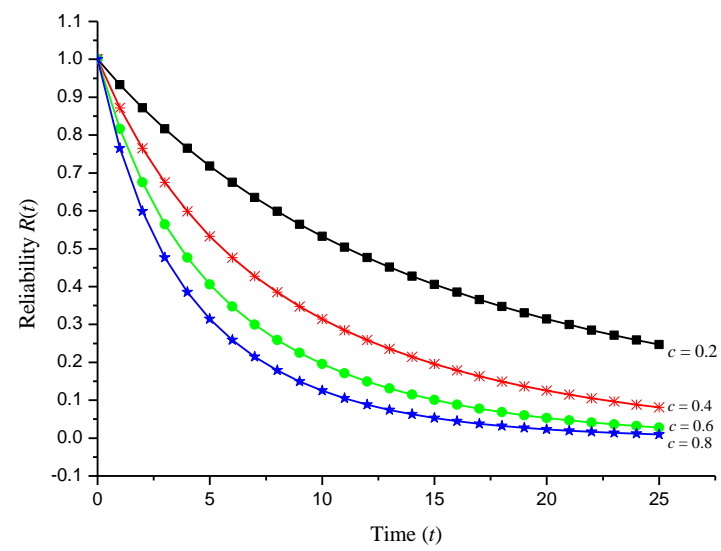

Figure 9. Reliability of the system with copula-coverage.

\subsection{Mean Time to Failure (MTTF) Analysis}

MTTF is the mean time to failure i.e., the time that the system is available or not failed. Taking $s$ tends to zero in reliability expression to get the MTTF of the system. 
International Journal of Mathematical, Engineering and Management Sciences

Vol. 6, No. 1, 322-344, 2021

https://doi.org/10.33889/IJMEMS.2021.6.1.021

\subsubsection{MTTF of the System without Copula- Coverage}

Taking all repair rate equal to zero and $s$ tends to zero, we get following MTTF expression without copula-coverage

$\operatorname{MTTF}_{1}=\frac{1}{2 \lambda_{1}+2 \lambda_{2}+\lambda_{m}+\lambda_{h}}\left[1+\frac{2 \lambda_{1}}{\lambda_{2}+\lambda_{h}}+\frac{2 \lambda_{2}}{\lambda_{1}+\lambda_{h}}\right]$

Now setting $\lambda_{1}=0.05, \lambda_{2}=0.10, \lambda_{h}=0.15, \lambda_{m}=0.20$ and varying $\lambda_{1}, \lambda_{2}, \lambda_{h}, \lambda_{m}$ one by one from 0.1 to 0.9 , we get the table of MTTF as Table 10 for $\lambda_{1}, \lambda_{2}, \lambda_{h}, \lambda_{m}$

Table 10. MTTF of the system without copula-coverage.

\begin{tabular}{ccccc}
\hline Failure rates & \multicolumn{3}{c}{ MTTF } \\
\cline { 2 - 5 }$\lambda_{1}, \lambda_{2}, \lambda_{h}, \lambda_{m}$ & $\lambda_{1}$ & $\lambda_{2}$ & $\lambda_{h}$ & $\lambda_{m}$ \\
0.1 & 3.466667 & 3.692308 & 4.166667 & 4.363636 \\
0.2 & 3.338346 & 3.865546 & 2.857143 & 3.692308 \\
0.3 & 3.342995 & 4.021164 & 2.187500 & 3.199999 \\
0.4 & 3.380471 & 4.145455 & 1.777778 & 2.823529 \\
0.5 & 3.424318 & 4.244032 & 1.500000 & 2.526316 \\
0.6 & 3.466667 & 4.323232 & 1.298701 & 2.285714 \\
0.7 & 3.505279 & 4.387918 & 1.145833 & 2.086957 \\
0.8 & 3.539779 & 4.441592 & 1.025641 & 1.920000 \\
0.9 & 3.570415 & 4.486772 & 0.928571 & 1.777778 \\
\hline
\end{tabular}

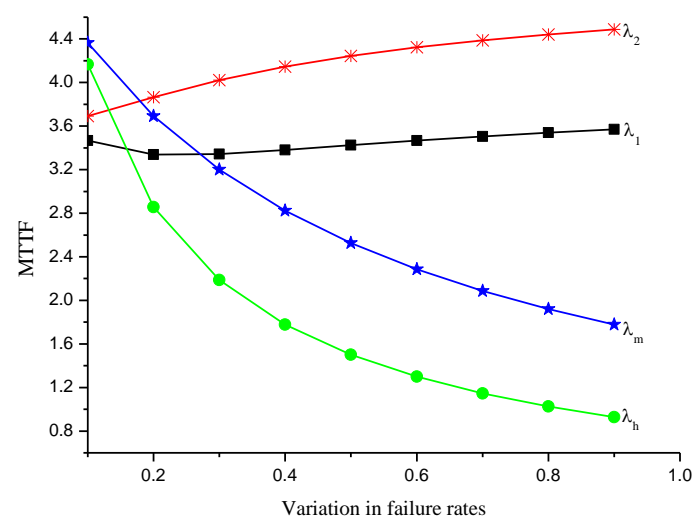

Figure 10. MTTF of the system without copula-coverage.

\subsubsection{MTTF of the System with Copula}

MTTF of the system with copula is given by the Equation (34). By setting $\lambda_{1}=0.05, \lambda_{2}=0.10, \lambda_{h}=$ $0.15, \lambda_{m}=0.20$ and varying $\lambda_{1}, \lambda_{2}, \lambda_{h}, \lambda_{m}$ one by one from 0.1 to 0.9 in Equation (34), we get the table of MTTF as Table 11 for $\lambda_{1}, \lambda_{2}, \lambda_{h}, \lambda_{m}$. 
International Journal of Mathematical, Engineering and Management Sciences

Vol. 6, No. 1, 322-344, 2021

https://doi.org/10.33889/IJMEMS.2021.6.1.021

Table 11. MTTF of the system with copula.

\begin{tabular}{ccccc}
\hline Failure rates & \multicolumn{3}{c}{ MTTF } \\
\cline { 2 - 5 }$\lambda_{1}, \lambda_{2}, \lambda_{h}, \lambda_{m}$ & $\lambda_{1}$ & $\lambda_{2}$ & $\lambda_{h}$ & $\lambda_{m}$ \\
0.1 & 3.466667 & 3.692308 & 4.166667 & 4.363636 \\
0.2 & 3.338346 & 3.865546 & 2.857143 & 3.692308 \\
0.3 & 3.342995 & 4.021164 & 2.187500 & 3.199999 \\
0.4 & 3.380471 & 4.145455 & 1.777778 & 2.823529 \\
0.5 & 3.424318 & 4.244032 & 1.500000 & 2.526316 \\
0.6 & 3.466667 & 4.323232 & 1.298701 & 2.285714 \\
0.7 & 3.505279 & 4.387918 & 1.145833 & 2.086957 \\
0.8 & 3.539779 & 4.441592 & 1.025641 & 1.920000 \\
0.9 & 3.570415 & 4.486772 & 0.928571 & 1.777778 \\
\hline
\end{tabular}

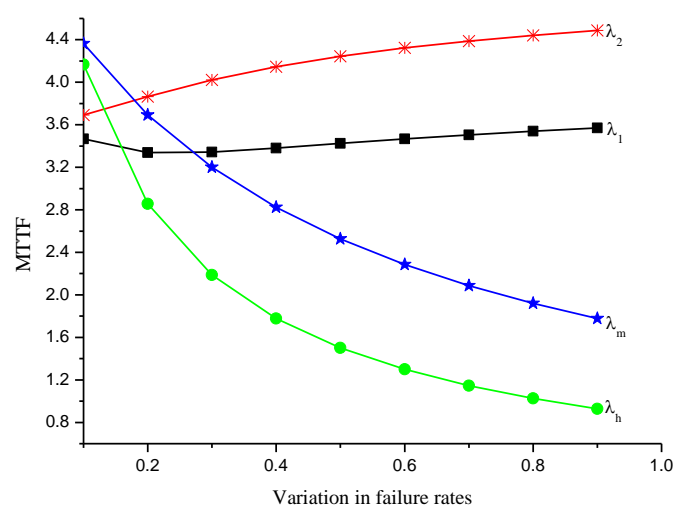

Figure 11. MTTF of the system with copula.

\subsubsection{MTTF of the System with Coverage.}

Taking all repair rate equal to zero and $s \rightarrow 0$ we get MTTF expression with coverage

$\mathrm{MTTF}_{2}=\frac{1}{2 c \lambda_{1}+2 c \lambda_{2}+c \lambda_{m}+c \lambda_{h}}\left[1+\frac{2 c \lambda_{1}}{c \lambda_{2}+c \lambda_{h}}+\frac{2 c \lambda_{2}}{c \lambda_{1}+c \lambda_{h}}\right]$

Now setting $\lambda_{1}=0.05, \lambda_{2}=0.10, \lambda_{h}=0.15, \lambda_{m}=0.20$ and varying $\lambda_{1}, \lambda_{2}, \lambda_{h}, \lambda_{m}$ one by one from 0.1 to 0.9 , we get the table of MTTF as Table 12 for $\lambda_{1}, \lambda_{2}, \lambda_{h}, \lambda_{m}$ at coverage values $c=0.2,0.4,0.6$, 0.8 .

Table 12. MTTF of the system with coverage.

\begin{tabular}{|c|c|c|c|c|c|c|c|c|c|c|c|c|c|c|c|c|}
\hline \multirow{3}{*}{$\begin{array}{l}\text { Failure } \\
\text { rates of } \\
\lambda_{1}, \lambda_{2}, \lambda_{h}, \\
\lambda_{m}\end{array}$} & \multicolumn{16}{|c|}{ MTTF } \\
\hline & \multicolumn{4}{|c|}{$c=0.2$} & \multicolumn{4}{|c|}{$c=0.4$} & \multicolumn{5}{|c|}{$c=0.6$} & \multicolumn{3}{|c|}{$c=0.8$} \\
\hline & $\lambda_{1}$ & $\lambda_{2}$ & $\lambda_{h}$ & $\lambda_{m}$ & $\lambda_{1}$ & $\lambda_{2}$ & $\lambda_{h}$ & $\lambda_{m}$ & $\lambda_{1}$ & $\lambda_{2}$ & $\lambda_{h}$ & $\lambda_{m}$ & $\lambda_{1}$ & $\lambda_{2}$ & $\lambda_{h}$ & $\lambda_{m}$ \\
\hline 0.1 & 17.33 & 18.46 & 20.83 & 21.81 & 8.666 & 9.231 & 10.41 & 10.90 & 5.777 & 6.153 & 6.944 & 7.272 & 4.333 & 4.615 & 5.208 & 5.454 \\
\hline 0.2 & 16.69 & 19.32 & 14.28 & 18.46 & 8.345 & 9.663 & 7.142 & 9.230 & 5.563 & 6.442 & 4.761 & 6.153 & 4.172 & 4.831 & 3.571 & 4.615 \\
\hline 0.3 & 16.71 & 20.10 & 10.93 & 16.00 & 8.357 & 10.05 & 5.468 & 7.999 & 5.571 & 6.701 & 3.645 & 5.333 & 4.178 & 5.026 & 2.734 & 4.000 \\
\hline 0.4 & 16.90 & 20.72 & 8.888 & 14.11 & 8.451 & 10.36 & 4.444 & 7.058 & 5.634 & 6.909 & 2.962 & 4.705 & 4.225 & 5.181 & 2.222 & 3.529 \\
\hline 0.5 & 17.12 & 21.22 & 7.500 & 12.63 & 8.560 & 10.61 & 3.750 & 6.315 & 5.707 & 7.073 & 2.500 & 4.210 & 4.280 & 5.305 & 1.875 & 3.157 \\
\hline 0.6 & 17.33 & 21.61 & 6.493 & 11.42 & 8.666 & 10.80 & 3.246 & 5.714 & 5.777 & 7.205 & 2.164 & 3.809 & 4.333 & 5.404 & 1.623 & 2.857 \\
\hline 0.7 & 17.52 & 21.93 & 5.729 & 10.43 & 8.763 & 10.96 & 2.864 & 5.217 & 5.842 & 7.313 & 1.909 & 3.478 & 4.381 & 5.484 & 1.432 & 2.608 \\
\hline 0.8 & 17.69 & 22.20 & 5.128 & 9.600 & 8.849 & 11.10 & 2.564 & 4.800 & 5.899 & 7.402 & 1.709 & 3.199 & 4.424 & 5.551 & 1.282 & 2.400 \\
\hline 0.9 & 17.85 & 22.43 & 4.642 & 8.888 & 8.926 & 11.21 & 2.321 & 4.444 & 5.951 & 7.4779 & 1.547 & 2.962 & 4.463 & 5.608 & 1.161 & 2.222 \\
\hline
\end{tabular}


International Journal of Mathematical, Engineering and Management Sciences

Vol. 6, No. 1, 322-344, 2021

https://doi.org/10.33889/IJMEMS.2021.6.1.021
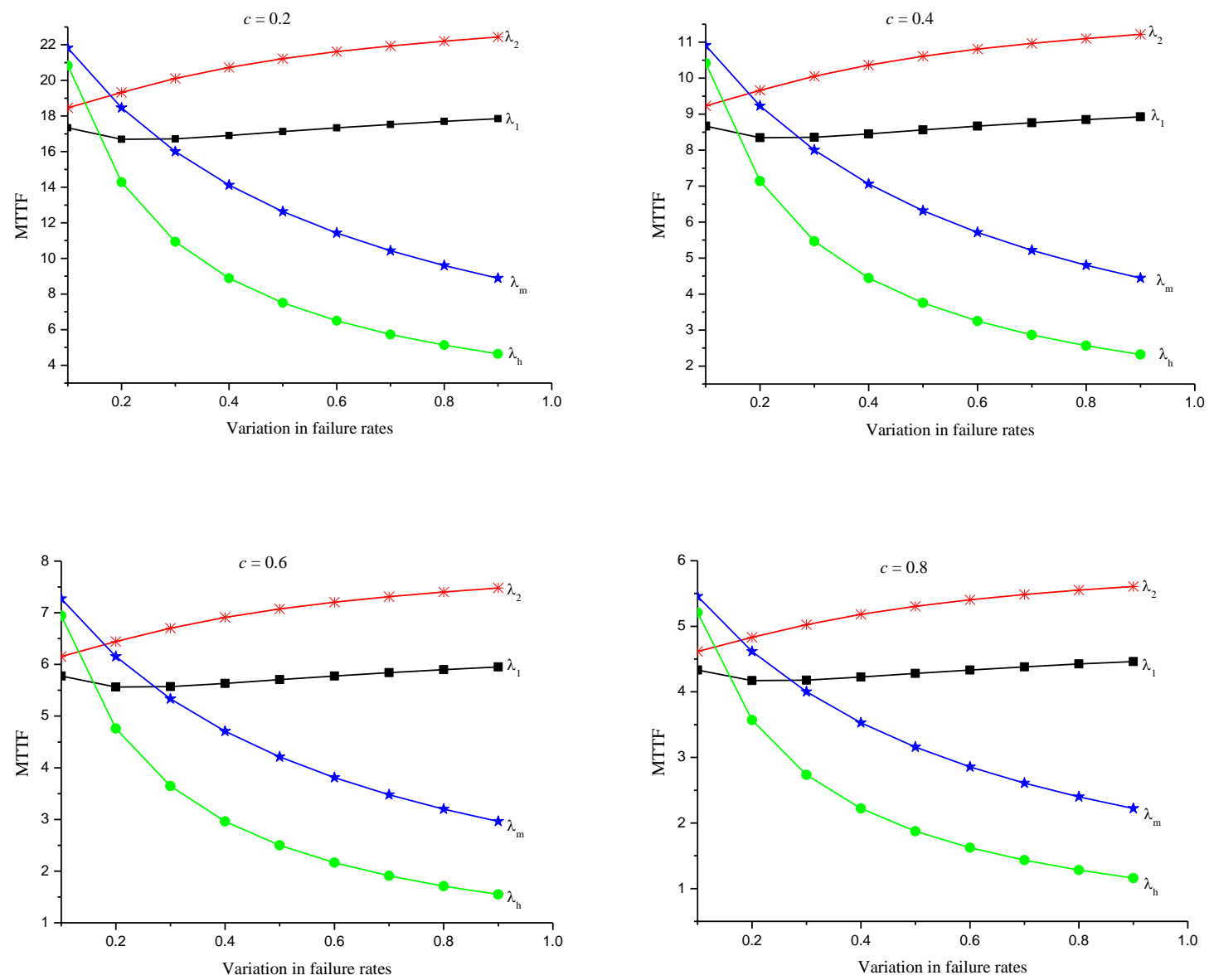

Figure 12. MTTF of the system with coverage.

\subsubsection{MTTF of the System with Copula- Coverage.}

MTTF with copula-coverage is given by Equation (35). By setting $\lambda_{1}=0.05, \lambda_{2}=0.10, \lambda_{h}=0.15$, $\lambda_{m}=0.20$ and varying $\lambda_{1}, \lambda_{2}, \lambda_{h}, \lambda_{m}$ one by one from 0.1 to 0.9 in Equation (35), we get the table of MTTF as Table 13 for $\lambda_{1}, \lambda_{2}, \lambda_{h}, \lambda_{m}$ at coverage values $c=0.2,0.4,0.6,0.8$.

Table 13. MTTF of the system with copula- coverage.

\begin{tabular}{|c|c|c|c|c|c|c|c|c|c|c|c|c|c|c|c|c|}
\hline \multirow{3}{*}{$\begin{array}{l}\text { Failure } \\
\text { rates of } \lambda_{1}, \\
\lambda_{2}, \lambda_{h}, \lambda_{m}\end{array}$} & \multicolumn{16}{|c|}{ MTTF } \\
\hline & \multicolumn{4}{|c|}{$c=0.2$} & \multicolumn{4}{|c|}{$c=0.4$} & \multicolumn{4}{|c|}{$c=0.6$} & \multicolumn{4}{|c|}{$c=0.8$} \\
\hline & $\lambda_{1}$ & $\lambda_{2}$ & $\lambda_{h}$ & $\lambda_{m}$ & $\lambda_{1}$ & $\lambda_{2}$ & $\lambda_{h}$ & $\lambda_{m}$ & $\lambda_{1}$ & $\lambda_{2}$ & $\lambda_{h}$ & $\lambda_{m}$ & $\begin{array}{l}\lambda_{1} \\
\end{array}$ & $\lambda_{2}$ & $\lambda_{h}$ & $\lambda_{m}$ \\
\hline 0.1 & 17.33 & 18.46 & 20.83 & 21.81 & 8.666 & 9.231 & 10.41 & 10.90 & 5.777 & 6.153 & 6.944 & 7.272 & 4.333 & 4.615 & 5.208 & 5.454 \\
\hline 0.2 & 16.69 & 19.32 & 14.28 & 18.46 & 8.345 & 9.663 & 7.142 & 9.230 & 5.563 & 6.442 & 4.761 & 6.153 & 4.172 & 4.831 & 3.571 & 4.615 \\
\hline 0.3 & 16.71 & 20.10 & 10.93 & 16.00 & 8.357 & 10.05 & 5.468 & 7.999 & 5.571 & 6.701 & 3.645 & 5.333 & 4.178 & 5.026 & 2.734 & 4.000 \\
\hline 0.4 & 16.90 & 20.72 & 8.888 & 14.11 & 8.451 & 10.36 & 4.444 & 7.058 & 5.634 & 6.909 & 2.962 & 4.705 & 4.225 & 5.181 & 2.222 & 3.529 \\
\hline 0.5 & 17.12 & 21.22 & 7.500 & 12.63 & 8.560 & 10.61 & 3.750 & 6.315 & 5.707 & 7.073 & 2.500 & 4.210 & 4.280 & 5.305 & 1.875 & 3.157 \\
\hline 0.6 & 17.33 & 21.61 & 6.493 & 11.42 & 8.666 & 10.80 & 3.246 & 5.714 & 5.777 & 7.205 & 2.164 & 3.809 & 4.333 & 5.404 & 1.623 & 2.857 \\
\hline 0.7 & 17.52 & 21.93 & 5.729 & 10.43 & 8.763 & 10.96 & 2.864 & 5.217 & 5.842 & 7.313 & 1.909 & 3.478 & 4.381 & 5.484 & 1.432 & 2.608 \\
\hline 0.8 & 17.69 & 22.20 & 5.128 & 9.600 & 8.849 & 11.10 & 2.564 & 4.800 & 5.899 & 7.402 & 1.709 & 3.199 & 4.424 & 5.551 & 1.282 & 2.400 \\
\hline 0.9 & 17.85 & 22.43 & 4.642 & 8.888 & 8.926 & 11.21 & 2.321 & 4.444 & 5.951 & 7.4779 & 1.547 & 2.962 & 4.463 & 5.608 & 1.161 & 2.222 \\
\hline
\end{tabular}



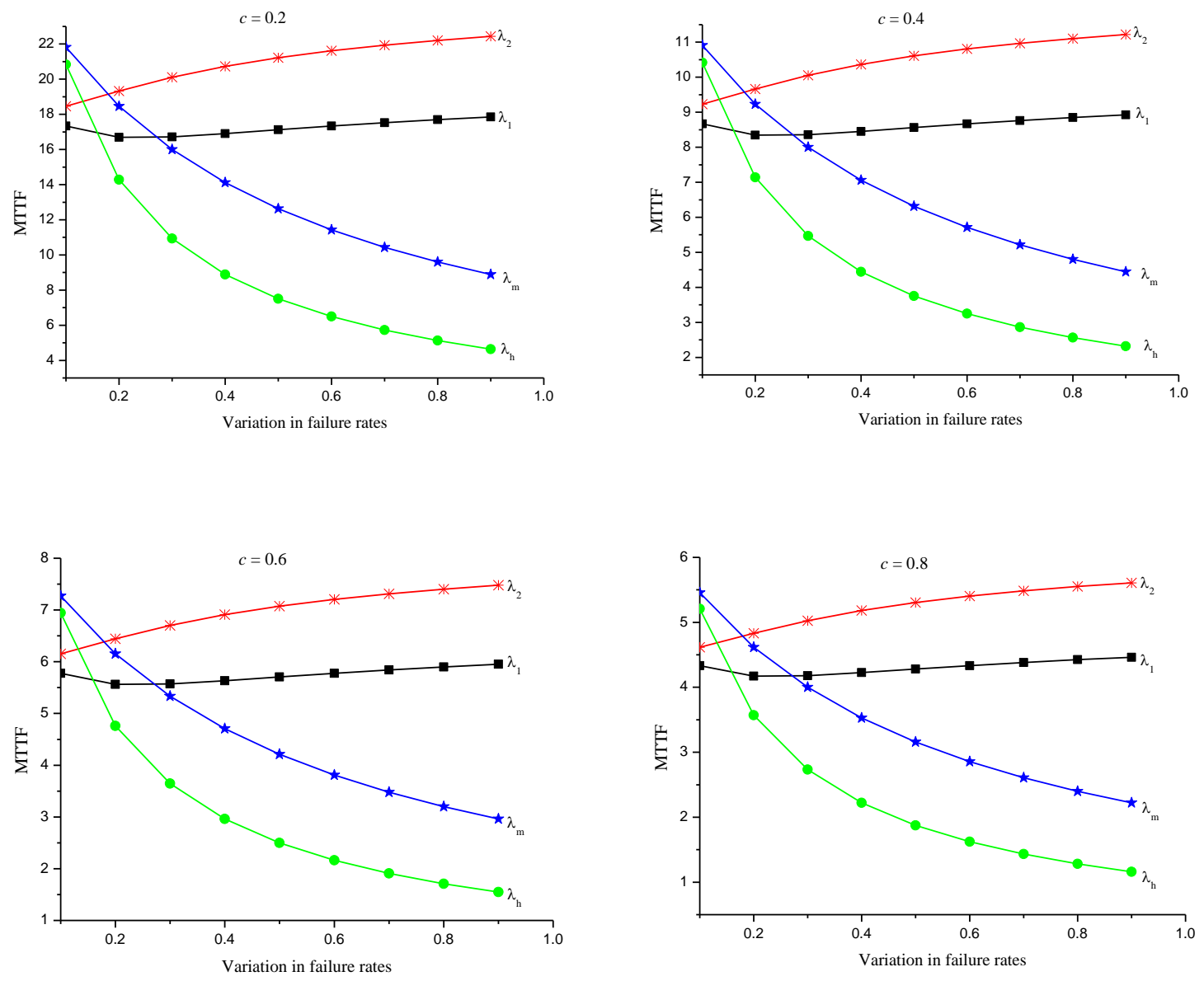

Figure 13. MTTF of the system with copula -coverage.

\section{Result Discussion}

From Figures 2-5, it is clearly shown that the availability of the system decreases slowly and it becomes constant after a long time, that is to say, that the availability of the system decreases as an increment in the time variable. During one type of repair between two transition states of the system (i.e., repair rate follows exponential distribution) and when no coverage technique applied, the availability of the system is achieved 0.786 as time varies from 0 to 25 units of time, which is shown by the Figure 2. While the availability of the system arising from Figure 3 is 0.909 as of time vary from 0 to 25 , Which is the result of availability when there is no coverage technique is applied and repair rate follows Gumbel-Hougaard family of copula distribution. Figure 4 shows the availability of the system at different values of coverage factor and when the repair rate follows an exponential distribution. With a close look of Figure 4, it is clear that availability of the system decreases with the passage of time and the highest value of availability is obtained at coverage value of 0.2 and lowest value of availability is obtained at coverage value of 0.8 , which is even better from the figure 2 and 3's results. Further, Figure 5 shows the availability of the system when both copula-coverage techniques applied together. With time availability of the system decreases up to 0.976 at coverage value of 0.2 and 0.92 at coverage value of 0.8 . From Figure 2-5, it can be 
International Journal of Mathematical, Engineering and Management Sciences

Vol. 6, No. 1, 322-344, 2021

https://doi.org/10.33889/IJMEMS.2021.6.1.021

easily seen that the availability is improved by using the mentioned techniques namely copula, coverage and copula-coverage. Among these techniques, the copula-coverage technique is the better one. The order of these techniques, to improve the availability of the system is copulacoverage $>$ coverage $>$ copula $>$ without copula-coverage.

The reliability's results of the system using the mentioned techniques are shown by figures 6-9. Figure 6 shows the reliability of the system when the repair rate follows exponential distribution and when the coverage technique is not applied. It is seen that as time increases the reliability of the system decreases. Figure 7 shows the reliability under the copula technique. As time increases, the reliability of the system decreases and it is up to 0.03 as time varies from 0 to 25 . Figure 8 demonstrates the reliability of the system using the coverage technique. At different values of coverage factor, the reliability of the system decreases with the increment in the time variable. Reliability is highest in case of coverage value 0.2 and lowest in case of coverage value 0.8 . Figure 9 shows the reliability when the copula and coverage technique applied together. The reliability of the system decreases as time increases at different values of coverage and it decreases up to 0.09 for the coverage value 0.8. It may be seen from Figures 6-9, the values of reliability are different under the copula, coverage and copula-coverage techniques. So, the copula-coverage technique must give the best value of reliability.

Table 10-13 gives the value of mean time to failure of the system and correspondingly Figures 1013 show the MTTF behaviour of the system. In all cases, MTTF variation is low for unit failure and high for human failure which indicates that human failure is more responsible for the proper functioning of the system. Also, it is to be noted that the coverage and copula-coverage techniques give the same values of MTTF. It means that there is no effect of copula technique on MTTF because MTTF does not depends on repair rate, either a single repair facility or more than one repair facility is available for the system.

\section{Conclusions and Future Work}

In this work, the authors proposed an approach for analysing the reliability measures of a parallel system consisting of two non-identical units, using Gumbel-Hougaard copula distribution and perfect coverage. A non-identical unit parallel system is often found in aircraft, medical equipment, power generating station, etc. Three different techniques namely copula, coverage and copulacoverage for improving the reliability of the system are discussed. The effect of copula, coverage, and copula-coverage, as well as the failure component of the system on to the behaviour of the system, is investigated by varying the different parameters of the system. Based on the results illustrated throughout this work, it is concluded that the availability and reliability of the system tend to become higher when copula and coverage techniques have been used together. The performance of the system, using these techniques is evaluated in descent order as follows: copulacoverage technique is better than the coverage technique, which is better than the copula technique. The comparative study of this work leads to the conclusion that, in order to improve system's reliability, there is a need to simultaneously incorporate copula and coverage techniques. Also, by preventing the occurrence of human failure, the system becomes more reliable. The area of this research is wide open in which one may investigate the behaviour of $k$-out-of- $n$ systems using these techniques. Also, this study can be more interesting if one may think about other types of copulas instead of the Gumbel-Hougaard family of copula. In future work, our research will look at repairing the degrade states and evaluating the sensitivity of availability, sensitivity of reliability and sensitivity of MTTF. 
International Journal of Mathematical, Engineering and Management Sciences

Vol. 6, No. 1, 322-344, 2021

https://doi.org/10.33889/IJMEMS.2021.6.1.021

\section{Conflict of Interest}

The authors declare that they have no conflict of interest.

\section{Acknowledgement}

The authors appreciate the effort from editors and reviewers. This paper did not receive any financial assistance/grants from any funding agencies. The authors are very thankful to the editor and reviewers for their time, suggestion and valuable comments.

\section{References}

Arnold, T.F. (1973). The concept of coverage and its effect on the reliability model of a repairable system. IEEE Transactions on Computers, 100(3), 251-254.

Arora, R., Tyagi, V., \& Ram, M. (2020). Multi-state system analysis with imperfect fault coverage, human error and standby strategies. Investigación Operacional, 4l(2), 214-231.

Chopra, G., \& Ram, M. (2019). Reliability measures of two dissimilar units parallel system using GumbelHougaard family copula. International Journal of Mathematical, Engineering and Management Sciences, 4(1), 116-130.

Dalah, C.M., \& Singh, V.V. (2014). Study of reliability measures of a two unit standby system under the concept of switch failure using copula distribution. American Journal of Computational and Applied Mathematics, 4(4), 118-129.

de Melo Mendes, B.V., \& de Souza, R.M. (2004). Measuring financial risks with copulas. International Review of Financial Analysis, 13(1), 27-45.

El-Sherbeny, M.S. (2013). Stochastic analysis of a two non-identical unit parallel system with different types of failures subject to preventive maintenance and repairs. Mathematical Problems in Engineering, 2013. Article ID 192545. Doi: 10.1155/2013/192545.

Genest, C., \& Favre, A.C. (2007). Everything you always wanted to know about copula modeling but were afraid to ask. Journal of Hydrologic Engineering, 12(4), 347-368.

$\mathrm{Hu}, \mathrm{L}$. (2006). Dependence patterns across financial markets: a mixed copula approach. Applied Financial Economics, 16(10), 717-729.

Levitin, G., \& Amari, S.V. (2007). Reliability analysis of fault tolerant systems with multi-fault coverage. International Journal of Performability Engineering, 3(4), 441-451.

Manglik, M., \& Ram, M. (2014). Stochastic modeling of a multi-state manufacturing system under three types of failures with perfect fault coverage. In Selected for Special issue in International Conference on Mathematical Techniques in Engineering Applications (ICMTEA 2013) at GEU, India with Journal of Engineering Science and Technology (pp. 77-90).

Nelsen, R.B. (2003, September). Properties and applications of copulas: A brief survey. In Proceedings of the First Brazilian Conference on Statistical Modeling in Insurance and Finance (pp. 10-28). University Press USP: São Paulo.

Nelsen, R.B. (2007). An introduction to copulas. Second addition. Springer Science \& Business Media, New York.

Niwas, R., \& Garg, H. (2018). An approach for analyzing the reliability and profit of an industrial system based on the cost free warranty policy. Journal of the Brazilian Society of Mechanical Sciences and Engineering, 40(5), 265. Doi: 10.1007/s40430-018-1167-8.

Patton, A.J. (2009). Copula-based models for financial time series. In Handbook of Financial Time Series (pp. 767-785). Springer, Berlin, Heidelberg. 
International Journal of Mathematical, Engineering and Management Sciences

Vol. 6, No. 1, 322-344, 2021

https://doi.org/10.33889/IJMEMS.2021.6.1.021

Pham, H. (1992). Reliability analysis of a high voltage system with dependent failures and imperfect coverage. Reliability Engineering \& System Safety, 37(1), 25-28.

Prabhudeva, S., \& Verma, A.K. (2007). Coverage modeling and reliability analysis using multi-state function. International Journal of Automation and Computing, 4(4), 380-387.

Ram, M., \& Goyal, N. (2018). Bi-directional system analysis under copula-coverage approach. Communications in Statistics-Simulation and Computation, 47(6), 1831-1844.

Ram, M., \& Singh, S.B. (2012). Cost benefit analysis of a system under head-of-line repair approach using Gumbel-Hougaard family copula. Journal of Reliability and Statistical Studies, 5(2), 105-118.

Ram, M., Singh, S.B., \& Singh, V.V. (2013). Stochastic analysis of a standby system with waiting repair strategy. IEEE Transactions on Systems, Man, and Cybernetics: Systems, 43(3), 698-707.

Reibman, A., \& Zaretsky, H. (1990, December). Modeling fault coverage and reliability in a fault-tolerant network. In [Proceedings] GLOBECOM'90: IEEE Global Telecommunications Conference and Exhibition (pp. 689-692). IEEE. San Diego, CA, USA.

Rodriguez, J.C. (2007). Measuring financial contagion: a copula approach. Journal of Empirical Finance, 14(3), 401-423.

Singh, V.V., \& Rawal, D.K. (2011). Availability analysis of a system having two units in series configuration with controller and human failure under different repair policies. International Journal of Scientific \& Engineering Research, 2(10), 1-9.

Sklar, A. (1973). Random variables, joint distribution functions, and copulas. Kybernetika, 9(6), 449-460.

Trivedi, P.K., \& Zimmer, D.M. (2007). Copula modeling: an introduction for practitioners. Now Publishers Inc, U.S.A.

Tyagi, V., Arora, R., Ram, M., \& Yadav, O.P. (2019). 2-Out-of-3: F System analysis under catastrophic failure. Nonlinear Studies, 26(3), 557-574.

Wang, K.H., Yen, T.C., \& Fang, Y.C. (2012). Comparison of availability between two systems with warm standby units and different imperfect coverage. Quality Technology \& Quantitative Management, 9(3), 265-282. 